Pacific Journal of Mathematics

CRITICAL VALUE SETS OF GENERIC MAPPING

J 


\title{
CRITICAL VALUE SETS OF GENERIC MAPPINGS
}

\author{
Goo Ishikawa, Satoshi KoIKe and Masahiro Shiota
}

Let $Y$ be a real analytic set. The subset of $Y$ consisting of all points where the local dimension of $Y$ is maximal is called the main part of $Y$. A subset $Y^{\prime}$ of a real analytic manifold $N$ is called a main semi-analytic set if $Y^{\prime}$ is the main part of some analytic set in a neighborhood of each point of $N$. In this paper it is shown that any proper $C^{\infty}$ mapping between analytic manifolds can be approximated by an analytic mapping in the Whitney topology so that the critical value set is a main semi-analytic set. An analogue holds true for the algebraic case too.

1. Analytic results. The topology of our spaces of $C^{\infty}$ or analytic mappings is the Whitney $C^{\infty}$ topology [see M. Hirsch [11] except for the last section, and $M, N$ always mean real analytic manifolds of dimension $n, p$ respectively. We denote by $\Sigma f$ the critical point set $\left\{x \in M \mid\right.$ rank $d f_{x}$ $<\min (n, p)\}$. Our main result is the following.

THEOREM 1. Let $f$ be a proper $C^{\infty}$ mapping from $M$ to $N$. Then every neighborhood of $f$ in $C^{\infty}(M, N)$ contains a proper analytic mapping $g$ such that $g(\Sigma g)$ is a main semi-analytic set of dimension $l=\min (n-1, p-1)$.

It is natural to ask if $g(\Sigma g)$ can be analytic in the above. The answer is negative.

EXAMPLE. Let $f: \mathbf{R}^{3} \rightarrow \mathbf{R}^{3}$ be the polynomial mapping defined by $f\left(x_{1}, x_{2}, x_{3}\right)=\left(x_{1}, x_{2}, x_{3}^{4}+x_{2} x_{3}^{2}+x_{1} x_{3}\right)$. Then $f(\Sigma f)$ is "Swallow's Tail" [see T. H. Bröcker [5] or M. Golubitsky and V. Guillemin [8]]. It is known that $f(\Sigma f)$ is not analytic. Moreover there exists a neighborhood $U$ of $f$ in $C^{\infty}\left(\mathbf{R}^{3}, \mathbf{R}^{3}\right)$ such that for any $g$ of $U, g(\Sigma g)$ is not an analytic set. (See Figure 1.)

We see easily that a main semi-analytic set is semi-analytic [see $\mathrm{S}$. Lojasiewicz [12]]. Any nowhere dense semi-analytic set is the critical value set of some analytic mapping, to say more precisely.

REMARK. Let $K$ be a semi-analytic subset of $N$ of codimension $>0$. Then there exist an analytic manifold $M$ and an analytic mapping $f$ : $M \rightarrow N$ such that

$$
\operatorname{dim} M=\operatorname{dim} N>\operatorname{dim} \Sigma f \text { and } f(\Sigma f)=K .
$$




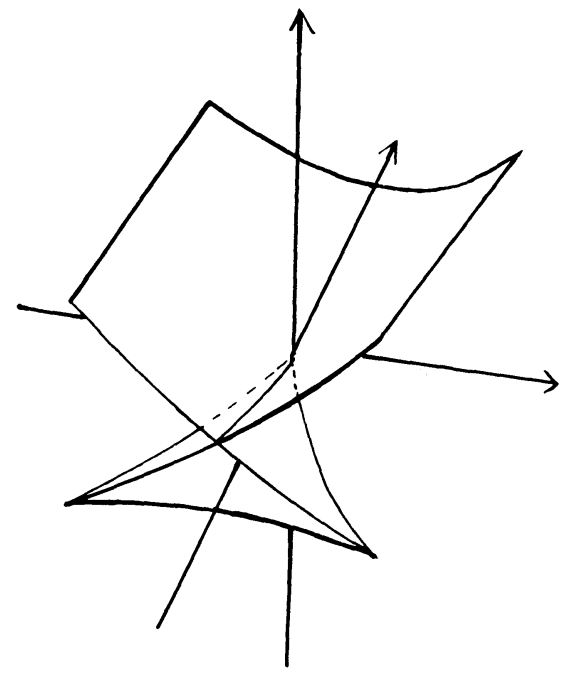

FIGURE 1

Let $Y$ be a main semi-analytic set of dimension $l$. Then we define the fundamental class of $Y$ in the homology group $H_{l}\left(Y ; \mathbf{Z}_{2}\right)$ as follows, where we use infinite chains if $Y$ is not compact. By [12] we have a triangulation of $Y$. Consider the homology groups of the simplicial complex with coefficient $\mathbf{Z}_{2}$. Then the sum of all $l$-simplexes defines a cycle, because any analytic set has the fundamental class [see A. Borel and A. Heafliger [4]]. The cycle is called the fundamental class of $Y$. If $Y$ is a subset of $N$, we denote by $[Y]$ the image of the fundamental class of $Y$ in $H_{l}\left(N ; \mathbf{Z}_{2}\right)$.

Proper $C^{\infty}$ mappings $f_{1}, f_{2}: M \rightarrow N$ are called proper homotopic if we have a proper $C^{\infty}$ mapping $F: M \times[0,1] \rightarrow N$ such that $\left.F\right|_{M \times 0}=f_{1}$ and $\left.F\right|_{M \times 1}=f_{2}$.

THEOREM 2. There exists an open dense subset $G$ of the set of all proper analytic mappings from $M$ to $N$ such

(i) for any $f$ of $G, f(\Sigma f)$ is a main semi-analytic set of dimension $l=\min (n-1, p-1)$,

(ii) the fundamental class of $\Sigma f$ is mapped to it of $f(\Sigma f)$ by $f_{*}$ for $f \in G$,

(iii) if $f$ and $g$ of $G$ are proper homotopic, we have $[f(\Sigma f)]=[g(\Sigma g)]$ in $H_{l}\left(N ; \mathbf{Z}_{2}\right)$.

2. Preliminaries. First, we prepare some notations of singularities [see J. Boardman [3] and J. Mather [14]]. For any integer $r \geq 1, J^{r}(n, p)$ is the linear space of $r$-jets of $C^{\infty}$ map germs $\left(\mathbf{R}^{n}, 0\right) \rightarrow\left(\mathbf{R}^{p}, 0\right)$, and $J^{r}(M, N)$ is the set of all $r$-jets of germs $(M, x) \rightarrow(N, y)$ for any $x \in M, y \in N$. 
Then $J^{r}(M, N)$ is fiber bundles over $M \times N$ and $M$ with fibers $J^{r}(n, p)$, $J^{r}(n, p) \times N$ respectively. We put for any integer $r>0$

$$
\begin{gathered}
M^{(k)}=\left\{\left(x_{1}, \ldots, x_{k}\right) \in M^{k}: x_{i} \neq x_{j} \text { for } i \neq j\right\}, \\
{ }_{k} J^{r}(M, N)=q^{-1}\left(M^{(k)}\right)
\end{gathered}
$$

where $q: J^{r}(M, N)^{k} \rightarrow M^{k}$ is the projection. For any $C^{\infty}$ mapping $f$ : $M \rightarrow N$, we denote by $j^{r} f$ the cross section of the fiber bundle $J^{r}(M, N) \rightarrow$ $M$ naturally defined by $f$, and by ${ }_{k} j^{r} f$ the restriction of $\left(j^{r} f\right)^{k}$ to $M^{(k)}$. If a subset $B$ of $J^{r}(n, p)$ is invariant under the coordinate transformations of $\left(\mathbf{R}^{n}, 0\right)$ and $\left(\mathbf{R}^{p}, 0\right)$, we denote by $B(M, N)$ the total space of the subbundle whose fiber is $B$, and by $B(f)$ the inverse image $j^{r} f^{-1}(B(M, N))$ for a $C^{\infty}$ mapping $f: M \rightarrow N$.

Let $\Sigma^{i}$ or $\Sigma^{i, J}$ denote the Thom-Boardman symbol (see J. Boardman [3]). We put

$$
B_{0}(f)= \begin{cases}\Sigma^{n-p+1,0}(f) & (n \geq p) \\ \Sigma^{1,0}(f) & (n<p) .\end{cases}
$$

We further put

$$
\Delta=\left\{\left(j^{2} f_{1}\left(x_{1}\right), j^{2} f_{2}\left(x_{2}\right)\right) \in_{2} J^{2}(M, N): f_{1}\left(x_{1}\right)=f_{2}\left(x_{2}\right)\right\} .
$$

Here we introduce the transversal condition concerning the Thom-Boardman singularities. We say that a $C^{\infty}$ mapping $f: M \rightarrow N$ satisfies the condition (T-B), if $f$ has the following properties:

(I) In the case $n \geq p$,

(1) $j^{1} f$ is transversal to each $\Sigma^{i}(M, N)$,

(2) $j^{2} f$ is transversal to each $\Sigma^{n-p+1, j}(M, N)$,

(3) ${ }_{2} j^{2} f$ is transversal to $\left(\Sigma^{n-p+1,0}(M, N) \times \Sigma^{n-p+1,0}(M, N)\right) \cap \Delta$.

(II) In the case $n<p$,

(1) $j^{1} f$ is transversal to each $\Sigma^{i}(M, N)$,

(2) $j^{2} f$ is transversal to each $\Sigma^{1, j}(M, N)$,

(3) ${ }_{2} j^{2} f$ is transversal to $\left(\Sigma^{1,0}(M, N) \times \Sigma^{1,0}(M, N)\right) \cap \Delta$.

3. Proof of Theorem 1. In this section we give the proof of Theorem 1.

Lemma 3.1. [Multi Transversality Theorem [8], [11], [14].] Let $\delta$ be a Whitney stratification of a closed subset of ${ }_{k} J^{r}(M, N)$. Then the set of $C^{\infty}$ mappings $f: M \rightarrow N$ such that ${ }_{k} j^{r} f$ is transversal to each stratum of $\mathcal{S}$ is dense in $C^{\infty}(M, N)$. 
A germ of $C^{\infty}$ mapping $f:(M, x) \rightarrow(N, f(x))$ is called $\mathcal{K}_{\text {-finite }}$, if the quotient $\mathcal{E}_{M, x} /\left(J_{f, x}+f_{x}^{*}\left(\mathfrak{K}_{N, f(x)}\right) \mathcal{E}_{M, x}\right)$ is finite dimensional over $\mathbf{R}$, where $J_{f, x}$ is the ideal in $\mathcal{E}_{M, x}$ generated by $(p \times p)$-minors of the Jacobian matrix of $f$ at $x$ and $\mathfrak{T}_{N, f(x)}$ is the maximal ideal of $\mathcal{E}_{N, f(x)}$. Especially, a germ is called $\mathcal{K}_{-} \nu$-finite, if the quotient is at most $\nu$ dimensional. The following lemma is an easy consequence from III, Theorem 7.2 in G. Gibson et al. [7] and Lemma 3.1.

LEMMA 3.2. For sufficiently large $\nu$, the set of $C^{\infty}$ mappings $f: M \rightarrow N$ such that the germ $f_{x}$ is $\mathcal{K}$ - $\nu$-finite for all $x \in M$ is an open dense subset of $C^{\infty}(M, N)$.

Lemma 3.3 [H. Whitney [19].] The set $C^{\omega}(M, N)$ of analytic mappings is dense in $C^{\infty}(M, N)$.

LEMMA 3.4. Let $g: M \rightarrow N$ be a proper analytic mapping which satisfies the condition (T-B). Then $g$ has the following properties:

(i) $B_{0}(g)$ is dense in $\Sigma g$.

(ii) There exists a semi-analytic subset $L \supset \Sigma g-B_{0}(g)$ such that $\operatorname{dim} L<\operatorname{dim} \Sigma g$, and $\left.g\right|_{\Sigma g-L}: \Sigma g-L \rightarrow g(\Sigma g-L)$ is an analytic isomorphism.

Proof. It follows from (1) and (2) of (T-B) that $B_{0}(g)$ is dense in $\Sigma g$, has dimension $p-1$, and the restriction of $g$ to it is an immersion. Put

$$
\begin{aligned}
L_{1}=\left\{y \in N: \text { there exist points } x_{1}, x_{2} \in B_{0}(g)\right. \\
\text { such that } \left.x_{1} \neq x_{2} \text { and } g\left(x_{1}\right)=g\left(x_{2}\right)=y\right\} .
\end{aligned}
$$

Since $g$ is a proper analytic mapping, $g\left(B_{0}(g)\right)$ and $L_{1}$ are semi-analytic. By (3) of (T-B), we have $\operatorname{dim} L_{1}<\operatorname{dim} g\left(B_{0}(g)\right)$. Putting $L_{2}=\Sigma g-$ $B_{0}(g), L_{2}$ is semi-analytic and $\operatorname{dim} L_{2}<\operatorname{dim} \Sigma g$. Here we put $L=$ $g^{-1}\left(L_{1}\right) \cup L_{2}$. Then (ii) follows.

LEMMA 3.5. Let $g: M \rightarrow N$ be a proper analytic mapping such that for any point $x$ of $M$, the germ of $g$ at $x$ is $\mathcal{K}$-finite. Suppose that $B_{0}(g)$ is dense in $\Sigma g$ and for an analytic subset $L$ of $\Sigma g$ with $\operatorname{dim} L<\operatorname{dim} \Sigma g,\left.g\right|_{\Sigma g-L}$ : $\Sigma g-L \rightarrow g(\Sigma g-L)$ is an analytic isomorphism. Then $g(\Sigma g)$ is main semi-analytic.

Note. From the assumption that $B_{0}(g)$ is dense in $\Sigma g$, we see that the local dimension of $g(\Sigma g)$ is constant. 
Proof. Since $g$ is proper and $\left.g\right|_{\Sigma g}$ is locally finite-to-one, $g(\Sigma g)$ is closed and $\left.g\right|_{\Sigma g}$ is finite-to-one. Hence, $g(\Sigma g)$ turns out to be main semi-analytic if we show that for any point $x$ of $\Sigma g$ the image by $\left.g\right|_{\Sigma g}$ of a neighborhood of $x$ in $\Sigma g$ is the main part of some analytic set in a neighborhood of $g(x)$.

Since the germ of $g$ at $x$ is analytic and $K_{\mathcal{C}}$-finite, there exists a representative $g_{\mathbf{C}}: U \rightarrow V$ of the complexification of $g$ such that $\left.g_{\mathbf{C}}\right|_{\Sigma g_{\mathbf{C}} \cap U}$ is proper and finite-to-one, where $U$ [resp. $V$ ] is an open neighborhood of $x$ [resp. $g(x)$ ] in a complexification $M_{\mathbf{C}}$ [resp. $N_{\mathbf{C}}$ ] of $M$ [resp. $N$ ] [see C. T. C. Wall [20] and H. Hironaka [10]]. Then, using the same argument as the proof of Lemma 1.1 in R. Benedetti and A. Tognoli [2], we can prove that $g(\Sigma g \cap U)$ is the main part of some analytic set in $V \in N$ if we take $U, V$ smaller, as follows:

We take a desingularization $\pi: X \rightarrow \Sigma g_{\mathrm{C}} \cap U$ and an irreducible component $Y$ of $X$ with $\operatorname{dim}_{\mathbf{R}} \pi(Y) \cap \Sigma g=\operatorname{dim}_{\mathbf{R}} \Sigma g$. Put $\sigma=g_{\mathbf{C}} \circ \pi$ : $Y \rightarrow \sigma(Y)\left(\subset V \subset N_{\mathrm{C}}\right)$. First we prove

$$
\operatorname{dim}[(\sigma(Y) \cap N)-g(\pi(Y) \cap(\Sigma g-L))]<\operatorname{dim} g(\Sigma g) .
$$

From a reason of dimension, a regular value of $\left.\pi\right|_{Y}$ is contained in $\Sigma g-L$. Thus, at a point of $Y, \sigma$ is isomorphic. Hence there exists a complex analytic subset $S^{\prime}$ of $Y$ with codimension $>0$ such that $S^{\prime} \supset$ $\left(\left.\pi\right|_{Y}\right)^{-1}(L)$ and $\left.\sigma\right|_{Y-S^{\prime}}$ is a local isomorphism. Then $S=\sigma^{-1}\left(\sigma\left(S^{\prime}\right)\right)$ is a complex analytic subset of codimension $>0$ in $Y$. As $Y$ is connected, $\sigma(Y-S)$ is connected. Furthermore, $\sigma$ is proper. Thus $\left.\sigma\right|_{Y-S}: Y \rightarrow S \rightarrow$ $\sigma(Y-S)$ is a covering of finite degree. We claim that this degree is odd. In fact, $(\pi(Y) \cap \Sigma g)-\pi(S) \neq \varnothing$ and for a point $y \in g((\pi(Y) \cap \Sigma g)$ $-\pi(S))=\sigma(Y-S) \cap g(\Sigma g-L), \sigma^{-1}(y)$ consists of a unique real point and several pairs of non-real conjugate points. This implies that $\#\left(\sigma^{-1}(y)\right)$ is odd. Now assume inequality $(*)$ does not hold. Then the difference

$$
(\sigma(Y-S) \cap N)-(g(\pi(Y)) \cap \Sigma g)
$$

has an element $y^{\prime}$. But we see that $\sigma^{-1}\left(y^{\prime}\right)$ consists of only several pairs of non-real conjugate points, and $\#\left(\sigma^{-1}\left(y^{\prime}\right)\right)$ is even for the element $y^{\prime} \in$ $\sigma(Y-S)$, which is a contradiction. We consider the analytic closure

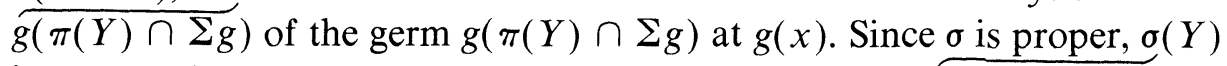
is a complex analytic subset of $V$ and $\sigma(Y) \cap N \supset \overparen{g(\pi(Y) \cap \Sigma g)}$ at $g(x)$. Thus, from $(*)$, we have

$(* *) \quad \operatorname{dim}[\overparen{g(\pi(Y)-\Sigma g)}-g(\pi(Y) \cap(\Sigma g-L))]<\operatorname{dim} g(\Sigma g)$

at $g(x)$. Lastly, we take a decomposition $X=\cup_{i} Y_{l}$ into a finite number of irreducible components. We denote by $A$ the union of $\overparen{g\left(\pi\left(Y_{i}\right) \cap \Sigma g\right)}$ 's. 
Then $A$ is an analytic set and contains $g(\Sigma g \cap U)$. Furthermore, from (**), $\operatorname{dim}(A-g(\Sigma g-L))<\operatorname{dim} g(\Sigma g)$ at $g(x)$. From Note, we have that $g(\Sigma g \cap U)$ is the main part of $A$. Thus Lemma 3.5 is proved.

Proof of Theorem 1. Let $f: M \rightarrow N$ be a proper $C^{\infty}$ mapping. From Lemmas 3.1-3.3 and the fact that the set of proper $C^{\infty}$ mappings is open in $C^{\infty}(M, N), f$ can be approximated by a proper analytic mapping $g$ : $M \rightarrow N$ such that for any $x$ of $M$, the germ of $g$ at $x$ is $\mathcal{K}$-finite, and $g$ satisfies (T-B). Hence, by Lemmas 3.4-3.5, we see that $g(\Sigma g)$ is main semi-analytic. This completes the proof of Theorem 1 .

\section{Proofs of the other results.}

Proof of the statement in Example. It is easy to check [see e.g. [7]] that this $f$ is stable in Mather's sence. Hence there exists a neighborhood $U$ of $f$ in $C^{\infty}\left(\mathbf{R}^{3}, \mathbf{R}^{3}\right)$ such that for any $g$ of $U$, we have $C^{\infty}$ diffeomorphisms $\tau_{1}, \tau_{2}$ of $\mathbf{R}^{3}$ such that $f=\tau_{1} \circ g \circ \tau_{2}$. Let $g \in U$. We want to see that $g(\Sigma g)$ is not an analytic set. Let $\tau$ be a $C^{\infty}$ diffeomorphism of $\mathbf{R}^{3}$ such that $\tau(f(\Sigma f))=g(\Sigma g)$. We assume $g(\Sigma g)$ to be analytic.

Now we see easily that the singular point set of $f(\Sigma f)$ contains $S_{1}=\left\{y_{1}=0, y_{2} \leq 0,4 y_{3}=-y_{2}^{2}\right\}$ where $\left(y_{1}, y_{2}, y_{3}\right)=f\left(x_{1}, x_{2}, x_{3}\right)$ and that $S_{2} \cap f(\Sigma f)=\{0\}$ where $S_{2}=\left\{y_{1}=0, y_{2} \geq 0,4 y_{3}=-y_{2}^{2}\right\}$. We can assume $\tau(0)=0$. Then the singular point set of $g(\Sigma g)$ contains $\tau\left(S_{1}\right)$. It is well-known that the singular point set of a semi-analytic set is semi-analytic [12]. Since $g(\Sigma g)$ is analytic, there exists a one-dimensional analytic set $S$ in a neighborhood $V$ of 0 such that

$$
g(\Sigma g) \supset S \supset \tau\left(S_{1}\right) \cap V .
$$

Let $h$ be an analytic function on $V$ such that $h^{-1}(0)=S$. As $S_{1}$ is diffeomorphic to $(-\infty, 0]$, there exists a $C^{\infty}$ imbedding $\phi:(-1,0] \rightarrow \mathbf{R}^{3}$ such that $\phi(0)=0, \phi((-1,0])=\tau\left(S_{1}\right) \cap V$. It follows that $h(\phi(t))=0$. Let $\psi=\left(\psi_{1}, \psi_{2}, \psi_{3}\right)$ be the Taylor expansion of $\phi$ at 0 . Then $\psi(t)$ is a formal series solution of the equation $h\left(y_{1}, y_{2}, y_{3}\right)=0$. By M. Artin Theorem [1], this equation has a convergent series solution $y(t)=$ $\left(y_{1}(t), y_{2}(t), y_{3}(t)\right)$ such that $y(t) \equiv \psi(t)$ modulo $\Re^{c}$ for any given integer $c$, where $\mathfrak{N}$ is the maximal ideal of the formal series ring. We see easily that the convergent solution is an analytic imbedding. This implies that $S$ is the image of $\psi$ in a neighborhood of 0 . Hence $S$ and $\tau\left(S_{2}\right)$ are not regularly situated at the origin [see [12] for the definition of "regularity situated"]. Therefore $S_{2}$ and $f(\Sigma f)$ are not regularly situated because of 
$\tau^{-1}(S) \subset f(\Sigma f)$. This contradicts the regular situation property of closed semi-analytic sets [12]. Hence $g(\Sigma g)$ is not analytic.

Proof of Theorem 2. Let $G^{\prime}$ be the set of proper analytic mappings $g$ : $M \rightarrow N$ such that for any $x \in M$, the germ of $g$ at $x$ is $\mathcal{K}_{-\nu}$-finite [ $\nu$ : sufficiently large], and $g$ satisfies (T-B). From the proof of Theorem 1, we see easily that $G^{\prime}$ includes an open dense subset $G$ of the set of proper analytic mappings, and (i) holds.

For any $f$ of $G$ we have a closed semi-analytic subset $K \subset \Sigma f$ such that $\Sigma f-K$ is an analytic manifold, the restriction of $f$ to which is an analytic imbedding, $\operatorname{dim} K<\operatorname{dim} \Sigma f, f^{-1} f(K)=K$, and $f(\Sigma f-K)$ is semi-analytic. By [12] there exist respective triangulations $L_{1}, L_{2}$ of $\Sigma f$, $f(\Sigma f)$, and subcomplexes $L_{1}^{\prime} \subset L_{1}, L_{2}^{\prime} \subset L_{2}$ such that $K$ and $f(K)$ correspond to the underlying polyhedrons of $L_{1}^{\prime}$ and $L_{2}^{\prime}$ respectively. Hence (ii) follows immediately.

From (ii), in order to prove (iii), it is sufficient to see $[\Sigma f]=[\Sigma g]$ in $H_{l}\left(M ; \mathbf{Z}_{2}\right)$. This follows from the fact that $j^{1} f$ and $j^{1} g$ are transversal to each $\Sigma^{i}(M, N)$ [see Theorem 7 in [16] for details of the proof].

5. Algebraic results. In this section we will consider algebraic analogues in the compact open $C^{\infty}$ topology. We assume this topology on any $C^{\infty}$ mappings space.

Let $Y$ be an algebraic set of $\mathbf{R}^{p}$. Then we see that the set $\{y \in Y$ : $\left.\operatorname{dim} Y_{y}=\operatorname{dim} Y\right\}$ is semi-algebraic [12]. We call this subset the main part of $Y$, and a semi-algebraic set of $\mathbf{R}^{p}$ is called main semi-algebraic if it is the main part of some algebraic set. We remark that for a main semi-algebraic set $Y^{\prime}$, the main part of the Zariski closure of $Y^{\prime}$ is $Y^{\prime}$. Here, we denote by $\overline{\bar{Y}^{\prime}}$ the Zariski closure of $Y^{\prime}$.

A $C^{\infty}$ algebraic manifold means at once an affine algebraic set and a $C^{\infty}$ manifold. Restrictions on a subset of polynomial mappings or rational mappings between Euclidean spaces are called equally polynomial or rational. A rational mapping of $C^{\infty}$ class is called a $C^{\infty}$ rational mapping.

THEOREM 3. Let $M\left[\subset \mathbf{R}^{m}\right]$ be a $C^{\infty}$ algebraic manifold, and $f$ : $M \rightarrow \mathbf{R}^{p}$ be a $C^{\infty}$ mapping. Then every neighborhood of $f$ contains a proper polynomial mapping $g: M \rightarrow \mathbf{R}^{p}$ such that the critical value set $g(\Sigma g)$ is main semi-algebraic.

REMARK. If $M$ is a closed $C^{\infty}$ manifold, any $C^{\infty}$ mapping $f: M \rightarrow \mathbf{R}^{p}$ can be approximated by one whose critical value set is main semi-algebraic. For any closed $C^{\infty}$ manifold is $C^{\infty}$ diffeomorphic to a $C^{\infty}$ algebraic manifold [see A. Tognoli [17]]. 
Proof of Theorem 3. By Weierstrass' polynomial approximation theorem, $f$ can be approximated by a polynomial mapping $g^{\prime \prime}: M \rightarrow \mathbf{R}^{p}$ [of degree $s$ ]. In this section, we take $s \geq 6$.

We denote by $P\left(\mathbf{R}^{m}, \mathbf{R}^{p}, l\right)$ the set of polynomial mappings $h: \mathbf{R}^{m} \rightarrow \mathbf{R}^{p}$ of degree at most $l$. Then $P\left(\mathbf{R}^{m}, \mathbf{R}^{p}, l\right)$ is identified with $\mathbf{R}^{u}$ naturally for some integer $u$. For a positive number $C>0$, let $g^{\prime}: \mathbf{R}^{m} \rightarrow \mathbf{R}^{p}$ be the polynomial mapping defined by $g^{\prime}(x)=\left(C|x|^{2 s}, \ldots, C|x|^{2 s}\right)$. Put

$$
Q=P\left(\mathbf{R}^{m}, \mathbf{R}^{p}, s\right)-\left\{h \in P\left(\mathbf{R}^{m}, \mathbf{R}^{p}, s\right): h+\left.g^{\prime}\right|_{M} \text { satisfies (T-B) }\right\} .
$$

LEMMA 5.1. $Q$ is a semi-algebraic set in $\mathbf{R}^{u}$ of codimension $>0$.

Proof. Let $F: M \times P\left(\mathbf{R}^{m}, \mathbf{R}^{p}, s\right) \rightarrow J^{1}\left(M, \mathbf{R}^{p}\right)$ be the mapping defined by $F^{\prime}(x, h)=j^{1}\left(h+\left.g^{\prime}\right|_{M}\right)(x), F^{\prime}: M \times P\left(\mathbf{R}^{m}, \mathbf{R}^{p}, s\right) \rightarrow J^{2}\left(M, \mathbf{R}^{p}\right)$ the mapping defined by $F^{\prime}(x, h)=j^{2}\left(h+\left.g^{\prime}\right|_{M}\right)(x)$, and $F^{\prime \prime}: M^{(2)} \times$ $P\left(\mathbf{R}^{m}, \mathbf{R}^{p}, s\right) \rightarrow{ }_{2} J^{2}\left(M, \mathbf{R}^{p}\right)$ the mapping defined by

$$
F^{\prime \prime}(x, h)={ }_{2} j^{2}\left(h+\left.g^{\prime}\right|_{M}\right)(x) \text {. }
$$

Then $F, F^{\prime}$, and $F^{\prime \prime}$ are onto submersions [see T. Fukuda [6]]. Using arguments given in [8], we easily see that $Q$ has measure zero in $\mathbf{R}^{u}$. By Tarski-Seidenberg Theorem, $Q$ is semialgebraic in $\mathbf{R}^{u}$, and $Q$ has codim $>$ 0 .

From Lemma 5.1, $g^{\prime \prime}$ can be approximated by a polynomial mapping $g=h+\left.g^{\prime}\right|_{M}$ which is proper and satisfies (T-B), where $h \in P\left(\mathbf{R}^{m}, \mathbf{R}^{p}, s\right)$. As $g$ is proper, $g(\Sigma g)$ is closed.

The next lemma follows similarly as Lemma 3.4.

LeMma 5.2. Let $g: M \rightarrow \mathbf{R}^{p}$ be a polynomial mapping which satisfies (T-B). Then $g$ has the following properties:

(i) $B_{0}(g)$ is dense in $\Sigma g$,

(ii) There exists a semi-algebraic subset $L \supset \Sigma g-B_{0}(g)$ such that $\operatorname{dim} L<\operatorname{dim} \Sigma g, \Sigma g-L$ is an analytic submanifold, and $\left.g\right|_{\Sigma g-L}: \Sigma g-L$ $\rightarrow g(\Sigma g-L)$ is an analytic isomorphism.

Lemma 5.3. Let $V$ be an algebraic subset of $\mathbf{R}^{s}$, and $\sigma: V \rightarrow \mathbf{R}^{t} a C^{\infty}$ rational mapping. Suppose $L$ is a semi-algebraic subset of $V$ such that $\operatorname{dim} L<\operatorname{dim} V$ and $\left.\sigma\right|_{V-L}: V-L \rightarrow \sigma(V-L)$ is an analytic isomorphism. Then there exists an algebraic subset $V^{\prime}$ of $\mathbf{R}^{t}$ such that $V^{\prime} \supset \sigma(V-L)$ and $\operatorname{dim}\left(V^{\prime}-\sigma(V-L)\right)<\operatorname{dim} V$.

For the proof see Lemma 1.1 in [2]. 
Applying Lemma 5.2 and Lemma 5.3, there exist a semi-algebraic subset $L \subset \Sigma g$ with $\operatorname{dim} L<\operatorname{dim} \Sigma g$, and an algebraic subset $V^{\prime}$ of $\mathbf{R}^{p}$ such that $V^{\prime} \supset g(\Sigma g-L)$ and $\operatorname{dim}\left(V^{\prime}-g(\Sigma g-L)\right)<\operatorname{dim} \Sigma g$. Since the closure of the set $\Sigma g-L$ is $\Sigma g$, we have $\overline{\overline{g(\Sigma g)}} \subset V^{\prime}$. Putting $S$ $=\overline{\overline{g(\Sigma g)}}-g(\Sigma g)$, we have $\operatorname{dim} S<\operatorname{dim} g(\Sigma g)$. Set

$$
C=\{y \in \overline{\overline{g(\Sigma g)}} \mid \operatorname{dim} \overline{\overline{g(\Sigma g)}} y=\operatorname{dim} \overline{\overline{g(\Sigma g)}}\} .
$$

For any $y$ of $C, S$ does not include $\overline{\overline{g(\Sigma g)}}$ as germs at $y$. Hence the germ of $\overline{\overline{g(\Sigma g)}}$ at $y$ and it of $g(\Sigma g)$ at $y$ intersect. Since $g(\Sigma g)$ is closed, we have $y \in g(\Sigma g)$. Thus we see that $g(\Sigma g)=C$, that is, $g(\Sigma g)$ is main semi-algebraic.

Let $M$ and $N$ be $C^{\infty}$ algebraic manifolds, and $f: M \rightarrow N$ a $C^{\infty}$ rational mapping. Then $f(\Sigma f)$ is a semi-algebraic subset of $N$.

Conversely, we have the following remark.

REMARK. Let $N$ be a $C^{\infty}$ algebraic manifold of dimension $n$, and $K$ a semi-algebraic subset of $N$ of codimension $>0$. Then there exist a $C^{\infty}$ algebraic manifold $M$ of dimension $n$ and a $C^{\infty}$ rational mapping $f$ : $M \rightarrow N$ with $\operatorname{dim} \Sigma f<n$ such that $f(\Sigma f)=K$.

\section{REFERENCES}

[1] M. Artin, On the solutions of analytic equations, Invent. Math., 5 (1968), 277-291.

[2] R. Benedetti and A. Tognoli, On real algebraic vector bundles, Bull. Sci. Math., $2^{e}$ série 104 (1980), 89-112.

[3] J. Boardman, Singularities of differentiable maps, Publ. Math. I. H. E. S., 33 (1967), 21-57.

[4] A. Borel and A. Heafliger, La classe d'homologie fondamentale d'un espace analytique, Bull. Soc. Math. France, 89 (1961), 461-513.

[5] T. H. Bröcker, Differentiable Germs and Catastrophes, London Math. Soc. Lecture Notes series, Cambridge Univ. Press.

[6] T. Fukuda, Local topological properties of differentiable mappings. I, Invent. Math., 65 (1981), 227-250.

[7] G. Gibson et al., Topological Stability of Smooth Mappings, Springer Lecture Notes 552, Berlin, 1976.

[8] M. Golubitsky and V. Guillemin, Stable Mappings and Their Singularities, Graduate texts in Math., 14, Springer Verlag, 1973.

[9] H. Hironaka, Resolution of singularities of algebraic variety, I-II, Ann. of Math., 79 (1964), 109-326.

[10] Stratification and Flatness, Real and Complex singularities, Oslo 1976, P. Holm Ed. Sijthoff and Noordhoff.

[11] M. Hirsch, Differential Topology, Springer Verlag, New York, 1976.

[12] S. Lojasiewicz, Ensembles semi-analytiques, I. H. E. S. Lecture Notes, 1965. 
[13] E. Looigenga, The discriminant of a real simple singularity, Comp. Math., 37 (1978), 51-62.

[14] J. Mather, Stability of $C^{\infty}$ mappings. V, Transversality, Advances in Math., 4 (1970), 301-336.

[15] B. Morin, Formes canoniques des singularités d'une application différentiable, Compt. Rend. Acad. Sci., Paris 260 (1965), 5662 - 5665, 6503 - 6506.

[16] R. Thom, Les singulatités des applications différentiables, Ann. Inst. Fourier, 6 (1956), $17-86$.

[17] A. Tognoli, Algebraic Geometry and Nash Functions, London and New York, Academic Press, 1977.

[18] D. J. A. Trotman, Stability of transversality to a stratification implies Whitney (a)-regularity, Invent. Math., 50 (1979), 273-277.

[19] H. Whitney, Analytic extensions of differentiable functions defined in closed sets, Trans. Amer. Math. Soc., 36 (1934), 63-89.

[20] C. T. C. Wall, Finite determinancy of smooth map-germs, Bull. London Math. Soc., 13 (1981), 481-539.

Received April 19, 1982 and in revised form June 9, 1983.

KYOTO UNIVERSITY

KYOTO, JAPAN

Hyogo University of Teacher Education

HYOGO, JAPAN

AND

KYOTO UNIVERSITY

KYOTO, JAPAN

Current address: Nagoya University

Nagoya, Japan 


\section{PACIFIC JOURNAL OF MATHEMATICS \\ EDITORS}

DONALD BABBITt (Managing Editor)

University of California

Los Angeles, CA 90024

J. Dugundu

University of Southern California

Los Angeles, CA 90089-1113

R. FINN

Stanford University

Stanford, CA 94305

HERMANN FLASCHKA

University of Arizona

Tucson, AZ 85721
C. C. MOORE

University of California

Berkeley, CA 94720

ARTHur Ogus

University of California

Berkeley, CA 94720

Hugo RossI

University of Utah

Salt Lake City, UT 84112

H. SAMELSON

Stanford University

Stanford, CA 94305

ASSOCIATE EDITORS

R. ARENS

E. F. BECKENBACH

B. H. NeumanN (1906-1982)

F. WOLF

K. YoshidA

\section{SUPPORTING INSTITUTIONS}

UNIVERSITY OF ARIZONA

UNIVERSITY OF BRITISH COLUMBIA

UNIVERSITY OF OREGON

CALIFORNIA INSTITUTE OF TECHNOLOGY

UNIVERSITY OF CALIFORNIA

MONTANA STATE UNIVERSITY

UNIVERSITY OF SOUTHERN CALIFORNIA

UNIVERSITY OF NEVADA, RENO

STANFORD UNIVERSITY

UNIVERSITY OF HAWAII

NEW MEXICO STATE UNIVERSITY

UNIVERSITY OF TOKYO

UNIVERSITY OF UTAH

OREGON STATE UNIVERSITY

WASHINGTON STATE UNIVERSITY

UNIVERSITY OF WASHINGTON 


\section{Pacific Journal of Mathematics}

Vol. 114, No. $1 \quad$ May, 1984

David Marion Arnold and Charles Irvin Vinsonhaler, Typesets and cotypesets of rank-2 torsion free abelian groups $\ldots \ldots \ldots \ldots \ldots \ldots \ldots 1$

Duncan Alan Buell and Richard Howard Hudson, Solutions of certain

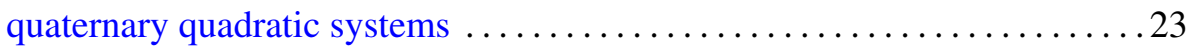

Hans Delfs and Manfred Knebusch, Separation, retractions and homotopy

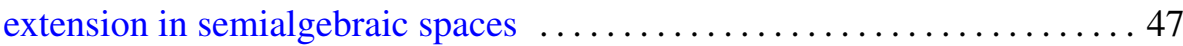

I. Erdélyi and Sheng-Wang Wang, A spectral duality theorem for closed

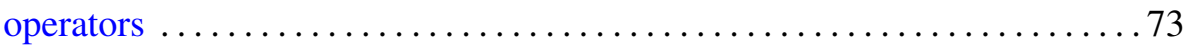

Theodore William Gamelin, Weak compactness of representing measures for $R(K)$

Kenneth R. Goodearl and T. H. Lenagan, Krull dimension of

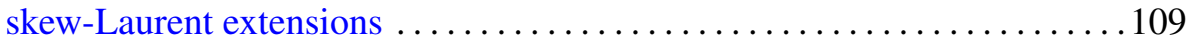

Daniel Joseph Gross, Compact quotients by $\mathbf{C}^{*}$-actions . . . . . . . . . . . 149

Goo Ishikawa, Satoshi Koike and Masahiro Shiota, Critical value sets of

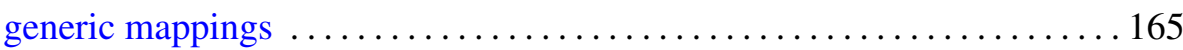

Hong Oh Kim, Derivatives of Blaschke products $\ldots \ldots \ldots \ldots \ldots \ldots \ldots \ldots \ldots$

Erhard Luft and Denis Karmen Sjerve, 3-manifolds with subgroups

$Z \oplus Z \oplus Z$ in their fundamental groups $\ldots \ldots \ldots \ldots \ldots \ldots \ldots \ldots \ldots \ldots \ldots \ldots$

George Clifford Nelson, Boolean powers, recursive models, and the Horn

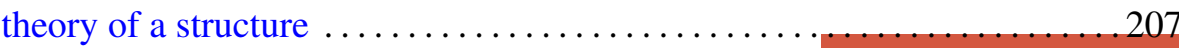

W. J. Phillips, Flow under a function and discrete decomposition of properly infinite $W^{*}$-algebras

Teodor C. Przymusiński, A solution to a problem of E. Michael ... 235

Bruce Harvey Wagner, Derivations of quasitriangular algebras 243 